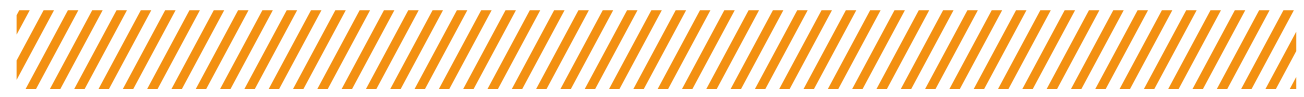

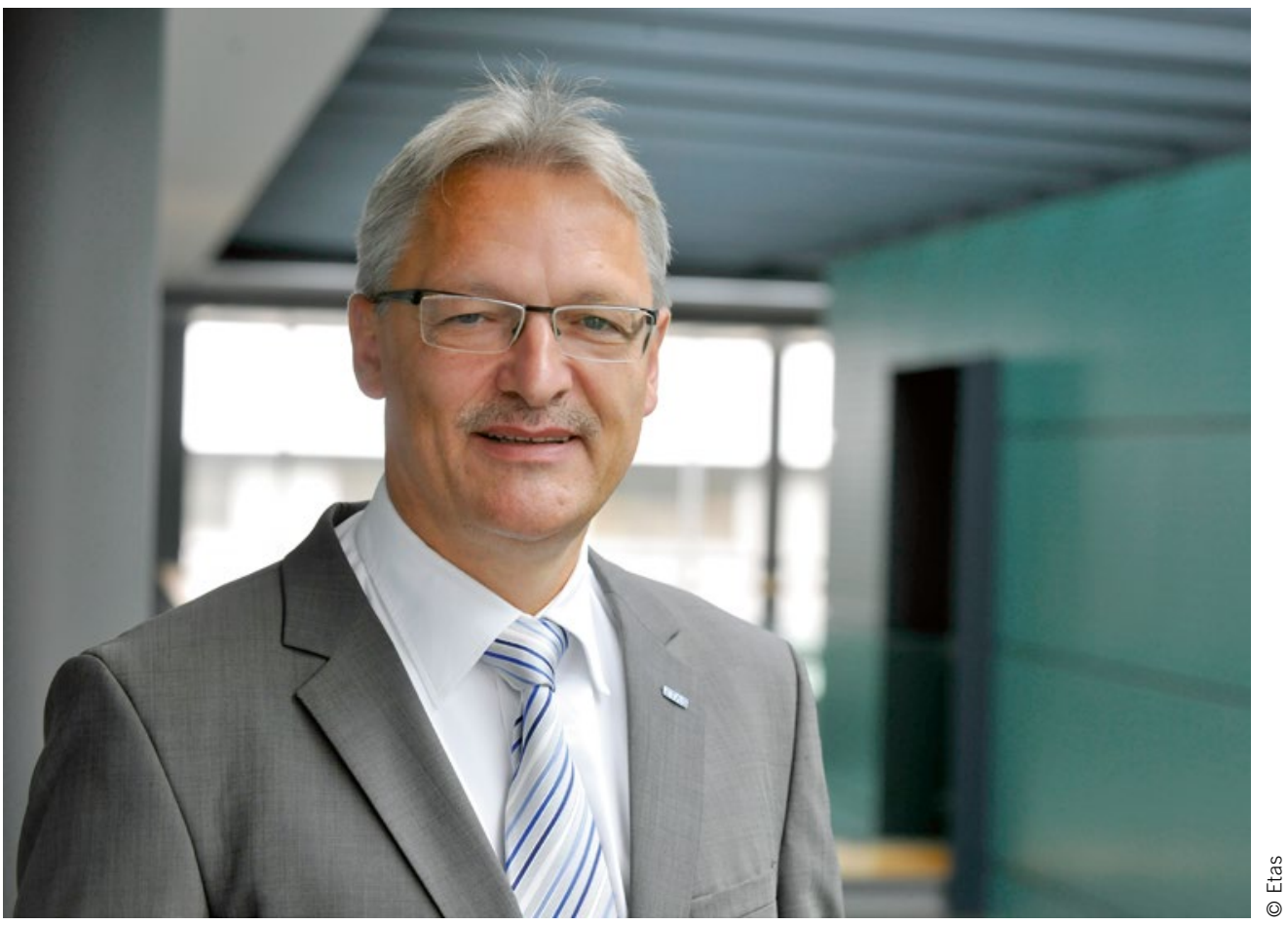

Friedhelm Pickhard

Chairman of the Board

of Management, Etas GmbH

\section{Measuring "Everything" Big Data in Automotive Engineering}

Big data refers to a statistical approach that seeks to obtain new information from huge amounts of data. The term is also used as a synonym for the efficient processing, management, and evaluation of complex data that exists in large quantities in a variety of forms and formats. Just as they yield benefits for other sections of the automobile industry, big data methods can also be a useful tool in automotive engineering.

In the past, isolated measurements were often carried out using spot checks in order to answer a single question. However, this approach falls short when it comes to predicting and mastering the behaviour of complex systems with the proper degree of certainty, systems such as modern high-tech IC engines, hybrid powertrains, and advanced braking and driver assistance systems. Here it is a big advantage to amalgamate all measurement tasks as much as possible; in other words, to measure "everything" at once in a set of tests carried out on the test bench or in the vehicle. This approach enables users to systematically reuse data, easily correlate different measurement variables, optimise dependent functions, and understand complex error patterns. At the same time, it facilitates the complete documentation of measurement results, such as for real driving emissions tests and OBD acceptance. There is also a compelling economic argument in favor of getting the maximum possible use out of test benches and test vehicles by integrating as many measurement tasks as possible into one set of tests.

The challenge this poses for in-vehicle measurement is the complete and time-synchronised recording of measurement signals from different sources. In the case of advanced driver assistance systems, for example, these sources include sensors such as stereo cameras and radar units, which supply a large amount of raw data in an uninterrupted stream during the journey data that has to be processed by the ECUs in real time.

The goal of using big data methods to shorten testing days and measurement campaigns is ambitious but realistic. The work done by calibration engineers will increasingly shift to virtual environments and to tasks carried out at their desks. Test drives will then serve only to corroborate the validations performed by means of simulations. And this no longer necessarily has to happen during a team's "own" test drive, but can also be based on measurement data gathered by other teams. To increase efficiency in the development process and master the complexity of electronic systems in vehicles, the use of big data methods is the next logical step. 